\title{
QUASILINEAR HYPERBOLIC EQUATIONS WITH HYSTERESIS
}

\author{
Augusto VISINTIN \\ Universitá degli Studi di Trento, Dipartimento di Matematica, via Sommarive 14, \\ 38050 Povo di Trento, Italy
}

Received 13 February 2001

ABSTRACT. - Plasticity, ferromagnetism, ferroelectricity and other phenomena lead to quasilinear hyperbolic equations of the form

$$
\frac{\partial^{2}}{\partial t^{2}}[u+\mathcal{F}(u)]+A u=f
$$

where $\mathcal{F}$ is a (possibly discontinuous) hysteresis operator, and $A$ is a second order elliptic operator. Existence of a solution is proved for an associated initial- and boundary-value problem in the framework of Sobolev spaces. The argument is based on the dissipation properties of hysteresis, and can be applied to a large class of hysteresis operators, including the classic Preisach model.

(C) 2002 L'Association Publications de l'Institut Henri Poincaré. Published by Elsevier B.V. All rights reserved

RÉSUMÉ. - Plusieurs phénomènes (plasticité, ferro-magnétisme, ferro-électricité, etc.) conduisent à la formulation d'une équation hyperbolique quasilinéaire de la forme

$$
\frac{\partial^{2}}{\partial t^{2}}[u+\mathcal{F}(u)]+A u=f
$$

ici $\mathcal{F}$ est un opérateur d'hystérésis éventuellement discontinu, et $A$ est un opérateur elliptique du deuxième ordre. On démontre l'existence d'une solution faible pour un problème aux limites et aux valeurs initiales associé à cette équation. L'argument est basé sur les propriétés de dissipation de l'hystérésis, et s'applique à une large classe d'opérateurs, qui inclut le modèle classique de Preisach.

(C) 2002 L'Association Publications de l'Institut Henri Poincaré. Published by Elsevier B.V. All rights reserved

\section{Introduction}

In the last years the mathematical research on hysteresis models has been progressing, see, e.g., $[1,4,6,10]$. In particular results have been obtained for PDEs containing hysteresis nonlinearities, including quasilinear parabolic and semilinear hyperbolic 
equations. On the other hand, if $\mathcal{F}$ is a scalar hysteresis operator and $A$ is a second order elliptic operator, the quasilinear hyperbolic equation

$$
\frac{\partial^{2}}{\partial t^{2}}[u+\mathcal{F}(u)]+A u=f
$$

has still been looking rather challenging, if we exclude the especially easy case in which $\mathcal{F}$ can be represented by one or more variational inequalities, see [10, Chap. VII]. In a single space dimension, existence of a solution of the initial- and boundary-value problem associated to (1) was proved by Krejčí $[5,6]$ assuming strict convexity of the hysteresis loops. Strict convexity is also required by known existence results for the analogous equation without hysteresis, see, e.g., Di Perna [3].

Although at first sight occurrence of hysteresis may look as an element of difficulty, its dissipative character suggests that it might allow to prove existence of a solution under weaker hypotheses than for the problem without hysteresis. This is shown in the present paper, in which existence of a solution is proved for the multidimensional problem, for a large class of (possibly discontinuous) nondegenerate scalar hysteresis operators. Discontinuities in the hysteresis relation account for the occurrence of free boundaries. Uniqueness of the solution remains an open question.

Equations of the form (1) occur in elastoplasticity, pseudoelasticity, ferromagnetism, ferrimagnetism, ferroelectricity, and in other physical phenomena; however, due to their scalar character, applications are essentially restricted to univariate systems. The study of Maxwell equations in three-dimensional domains requires the use of a vector hysteresis model. In [12] the hysteresis relation has been represented by a vector model of [2]; a weak formulation has been provided along the lines of the present paper, and existence of a solution has been proved for corresponding quasilinear hyperbolic and parabolic initial- and boundary-value problems, which are respectively obtained by including or neglecting displacement currents; see [14].

The plan of this paper is as follows. In Section 1 we outline the problem. In Section 2 we shortly illustrate how equations of the form (1) arise in mathematical physics. In Section 3 we provide a precise definition of the relay and Preisach operators. In Sections $4,5(6,7$, respectively) we formulate our problem for $A:=-\Delta$, and prove existence of a solution for $\mathcal{F}$ equal to a relay operator (a Preisach operator, resp.). In Section 8 we discuss a regularity issue, and deal with a modified formulation in which the variable $u$ is convoluted in space with a regularizing kernel. Finally, in Section 9 we point out some remarks, draw conclusions and discuss some open questions.

The results of the present paper have been announced in [13].

\section{The problem}

Hysteresis. A causal operator $\mathcal{F}$ which acts between Banach spaces of timedependent functions is called a hysteresis operator whenever it is rate-independent, that is, $[\mathcal{F}(u)](\varphi(t))=[\mathcal{F}(u \circ \varphi)](t)$ for any increasing time-homeomorphism $\varphi$ and for any instant $t$. We allow $\mathcal{F}$ to be multi-valued, and extend it to space- and time-dependent functions by setting $[\mathcal{F}(u)](x, t):=[\mathcal{F}(u(x, \cdot))](t)$; see [10] for details. 


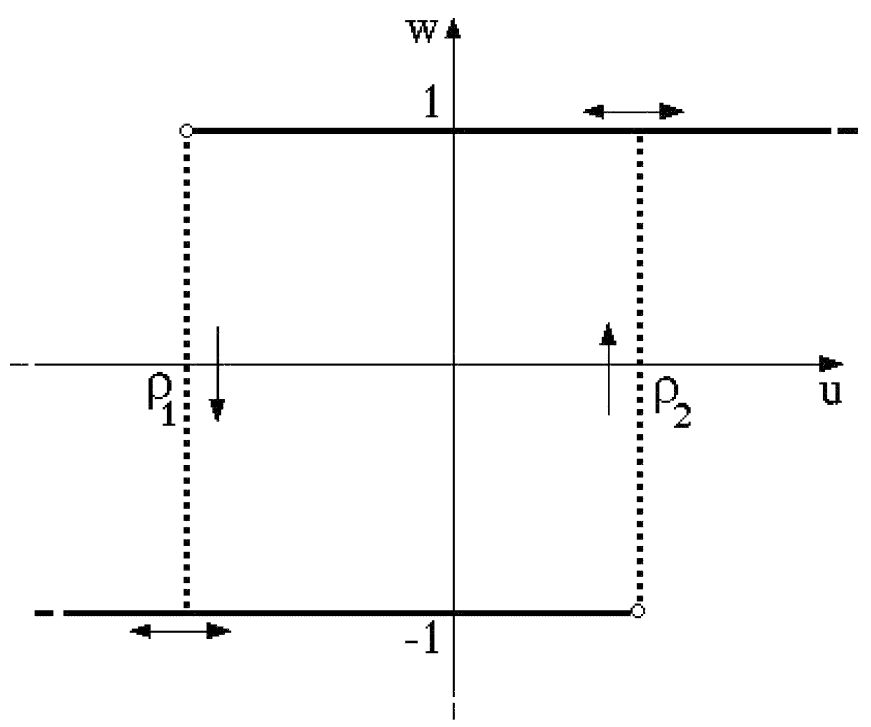

Fig. 1. Relay operator.

A large and important class of hysteresis operators is provided by the classic Preisach model; loosely speaking, this consists in a linear combination of more elementary operators, named (delayed) relay operators. Throughout this paper by $\rho$ we denote any pair $\left(\rho_{1}, \rho_{2}\right) \in \mathbf{R}^{2}$ such that $\rho_{1}<\rho_{2}$; the corresponding relay operator $h_{\rho}$ is outlined in Fig. 1. For instance, let $u(0)<\rho_{1}$; then $w(0)=-1$, and $w(t)=-1$ as long as $u(t)<\rho_{2}$; if at some instant $u$ reaches $\rho_{2}$ then $w$ jumps up to 1 , where it remains as long as $u(t)>\rho_{1}$; if later $u$ reaches $\rho_{1}$, then $w$ jumps down to -1 , and so on. Whenever $\rho_{1}<u(0)<\rho_{2}$, the initial value $w^{0}(= \pm 1)$ must be prescribed. The operator $h_{\rho}$ is causal and rate-independent; it is the most simple model of discontinuous hysteresis.

Let us now consider a finite Borel measure $\mu$ (called Preisach measure) on the half-plane $\mathcal{P}:=\left\{\rho:=\left(\rho_{1}, \rho_{2}\right) \in \mathbf{R}^{2}: \rho_{1}<\rho_{2}\right\}$ of admissible thresholds, and define the Preisach operator $\mathcal{H}_{\mu}:=\int_{\mathcal{P}} h_{\rho} \mathrm{d} \mu(\rho)$. See Fig. 2 for a simple example.

Let $\Omega$ be a bounded domain of $\mathbf{R}^{N}(N \geqslant 1)$ of Lipschitz class, $T>0$ and set $Q:=\Omega \times] 0, T[$. Let $f: Q \rightarrow \mathbf{R}$ be a given function, $\mathcal{F}$ a (possibly multi-valued) scalar hysteresis operator, and $A$ a second order elliptic operator; regularity requirements will be specified afterwards. In this paper we deal with the second order quasilinear hyperbolic equation

$$
\frac{\partial^{2}}{\partial t^{2}}[u+\mathcal{F}(u)]+A u=f \quad \text { in } Q,
$$

coupled with the initial and boundary conditions

$$
\begin{gathered}
u+w=u^{0}+w^{0}, \quad \frac{\partial}{\partial t}(u+w)=u^{1}+w^{1} \quad \text { in } \Omega \times\{0\}, \\
u=0 \quad \text { on } \partial \Omega \times] 0, T[
\end{gathered}
$$




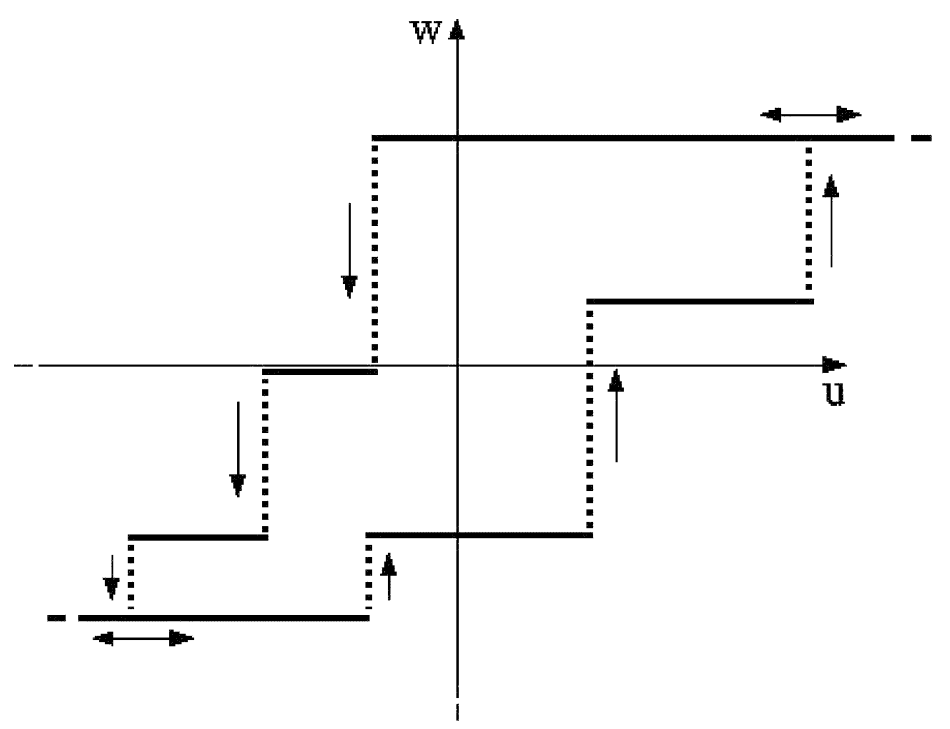

Fig. 2. Preisach model corresponding to a positive measure $\mu$ concentrated at three points of $\mathcal{P}$. here $u^{0}, w^{0}, u^{1}, w^{1}: \Omega \rightarrow \mathbf{R}$ are prescribed functions. Integrating (1.1) in time we get

$$
\frac{\partial}{\partial t}[u+\mathcal{F}(u)]+A \int_{0}^{t} u(\cdot, \tau) \mathrm{d} \tau=u^{1}+w^{1}+\int_{0}^{t} f(\cdot, \tau) \mathrm{d} \tau=: F \quad \text { in } Q .
$$

If the operator $A$ is in divergence form, Eq. (1.1) is also equivalent to a first-order system.

\section{Applications}

In this section we briefly illustrate how equations like (1.1) arise in continuum mechanics and in electromagnetism.

Elastoplasticity and pseudoelasticity. Let $\Omega \subset \mathbf{R}^{3}$ represent a continuum body, and let us denote the displacement vector by $u$, the (linearized) strain tensor by $\varepsilon$, the stress tensor by $\sigma$, and a distributed load by $h$. Newton's law and the definition of $\varepsilon$ yield

$$
\frac{\partial^{2} u_{i}}{\partial t^{2}}=\sum_{\ell=1}^{3} \frac{\partial \sigma_{i \ell}}{\partial x_{\ell}}+h_{i}, \quad \varepsilon_{i j}:=\frac{1}{2}\left(\frac{\partial u_{i}}{\partial x_{j}}+\frac{\partial u_{j}}{\partial x_{i}}\right) \quad \text { in } Q(i, j=1,2,3),
$$

whence

$$
\frac{\partial^{2} \varepsilon_{i j}}{\partial t^{2}}=\frac{1}{2} \sum_{\ell=1}^{3}\left(\frac{\partial^{2} \sigma_{i \ell}}{\partial x_{\ell} \partial x_{j}}+\frac{\partial^{2} \sigma_{j \ell}}{\partial x_{\ell} \partial x_{i}}\right)+\frac{1}{2}\left(\frac{\partial h_{i}}{\partial x_{j}}+\frac{\partial h_{j}}{\partial x_{i}}\right) \quad \text { in } Q(i, j=1,2,3) .
$$

In an elastoplastic material the dependence of the stress on the strain exhibits hysteresis. The same applies to austenitic materials exhibiting so-called pseudoelasticity, i.e., shape 
memory. In either case we may assume that $\varepsilon=a \sigma+\mathcal{F}_{1}(\sigma), a$ being a positive constant and $\mathcal{F}_{1}$ a tensor hysteresis operator. This yields an equation of the form (1.1) for tensor variables; for univariate systems, this is reduced to an equation for scalar variables.

Ferromagnetism and ferroelectricity. ${ }_{\vec{B}}$ Let $\Omega$ represent an electromagnetic material, and let us denote the electric field by $\vec{E}$, the electric displacement by $\vec{D}$, the electric current density by $\vec{J}$, the magnetic field by $\vec{H}$, the magnetic induction by $\vec{B}$, the electric conductivity by $\sigma$, the dielectric permittivity by $\varepsilon$, the magnetic permeability by $\mu$, the speed of light in vacuum by $c$, and an applied electromotive force by $\vec{g}$. For the sake of simplicity, here we assume that the equations in $\Omega$ can be decoupled from those outside. However, this outline and the results of this paper can be extended to the case in which this restriction is dropped.

In Gauss units, the Ampère, Faraday and Ohm laws respectively read

$$
\begin{gathered}
c \nabla \times \vec{H}=4 \pi \vec{J}+\frac{\partial \vec{D}}{\partial t} \quad \text { in } Q(\nabla \times:=\text { curl }), \\
c \nabla \times \vec{E}=-\frac{\partial \vec{B}}{\partial t} \quad \text { in } Q, \\
\vec{J}=\sigma(\vec{E}+\vec{g}) \quad \text { in } Q .
\end{gathered}
$$

In a ferromagnetic material we can assume that $\vec{D}=\varepsilon \vec{E}$. Applying the curl operator to (2.3), differentiating (2.4) in time, and eliminating $\vec{J}, \vec{D}, \vec{E}$, we then get

$$
\varepsilon \frac{\partial^{2} \vec{B}}{\partial t^{2}}+4 \pi \sigma \frac{\partial \vec{B}}{\partial t}+c^{2} \nabla \times \nabla \times \vec{H}=4 \pi c \sigma \nabla \times \vec{g} \quad \text { in } Q .
$$

This equation applies to both ferromagnetic and ferrimagnetic materials. The former are all metals, and so for slow processes the displacement term $\varepsilon \frac{\partial^{2} \vec{B}}{\partial t^{2}}$ is dominated by the Ohmic term $4 \pi \sigma \frac{\partial \vec{B}}{\partial t}$. In this case (2.6) is then usually replaced by the so-called $e d d y$ current equation

$$
4 \pi \sigma \frac{\partial \vec{B}}{\partial t}+c^{2} \nabla \times \nabla \times \vec{H}=4 \pi c \sigma \nabla \times \vec{g} \quad \text { in } Q .
$$

However, for fast processes (2.6) applies. On the other hand, ferrimagnetic materials may be insulators; (2.6) with $\sigma=0$ can then be used for either slow or fast processes.

In any of these cases $\vec{B}=\vec{H}+4 \pi \vec{M}$, and the magnetization $\vec{M}$ depends with hysteresis on $\vec{H}$. We may then assume that $\vec{M}=\mathcal{F}_{2}(\vec{H})$, where $\mathcal{F}_{2}$ is a vector hysteresis operator.

If instead we deal with a ferroelectric material, we may assume that $\vec{B}=\mu \vec{H}$; Eqs. (2.3), (2.4) and (2.5) then yield

$$
\mu \frac{\partial^{2} \vec{D}}{\partial t^{2}}+4 \pi \mu \sigma \frac{\partial \vec{E}}{\partial t}+c^{2} \nabla \times \nabla \times \vec{E}=-4 \pi \mu \sigma \frac{\partial \vec{g}}{\partial t} \quad \text { in } Q .
$$


Here $\vec{D}=\vec{E}+4 \pi \vec{P}$, and the electric polarization $\vec{P}$ depends on $\vec{E}$ with hysteresis; we may then assume that $\vec{P}=\mathcal{F}_{3}(\vec{E})$, where $\mathcal{F}_{3}$ is a vector hysteresis operator.

Special geometries. For univariate systems we are reduced to an equation for scalar variables, with $A=-\frac{\partial^{2}}{\partial x^{2}}$. Under severe restrictions on the geometry and on the symmetry of the fields, the above setting can also be reduced to a planar problem for scalar variables. Let $D$ be a domain of $\mathbf{R}^{2}, \Omega:=D \times \mathbf{R}$, and assume that, using orthogonal Cartesian coordinates $x, y, z, \vec{H}$ is parallel to the $z$-axis and only depends on the coordinates $x, y$, i.e., $\vec{H}=(0,0, H(x, y))$. Then

$$
\nabla \times \nabla \times \vec{H}=\left(0,0,-\Delta_{x, y} H\right) \quad\left(\Delta_{x, y}:=\frac{\partial^{2}}{\partial x^{2}}+\frac{\partial^{2}}{\partial y^{2}}\right) \quad \text { in } \Omega .
$$

Dealing with a strongly anysotropic material, we can also assume that $\vec{M}=(0,0$, $M(x, y))$. If $\nabla \times \vec{g}:=(0,0, r)$, Eq. (2.6) is then reduced to

$$
\varepsilon \frac{\partial^{2}}{\partial t^{2}}(H+4 \pi M)+4 \pi \sigma \frac{\partial}{\partial t}(H+4 \pi M)-c^{2} \Delta_{x, y} H=4 \pi c \sigma r \text { in } D .
$$

We can then assume that $M=\mathcal{F}(H)$, where $\mathcal{F}$ is a scalar hysteresis operator. A similar discussion applies to (2.8).

Remark. - We have represented the above phenomena by equations to the form (1.1). This has required differentiation operations, and indeed (1.1) can be expected to hold just in the sense of distributions. We might also derive equivalent systems. For instance, in continuum mechanics one might just couple the system (2.1) with the constitutive law $\varepsilon=a \sigma+\mathcal{F}_{1}(\sigma)$. Similarly, in electromagnetism one might couple the Maxwell-Ohm equations (2.3), (2.4), (2.5) with the appropriate constitutive relations.

\section{Hysteresis}

In this section we make the definition of the relay and Preisach operators precise, and specify the functional framework.

Relay operator. Let us fix any pair $\rho:=\left(\rho_{1}, \rho_{2}\right) \in \mathbf{R}^{2}, \rho_{1}<\rho_{2}$. For any $u \in$ $C^{0}([0, T])$ and any $\xi \in\{-1,1\}$, we set $\left.X_{t}:=\{\tau \in] 0, t\right]: u(\tau)=\rho_{1}$ or $\left.\rho_{2}\right\}$ and define the function $w=h_{\rho}(u, \xi):[0, T] \rightarrow\{-1,1\}$ as follows:

$$
\begin{gathered}
w(0):= \begin{cases}-1 & \text { if } u(0) \leqslant \rho_{1}, \\
\xi & \text { if } \rho_{1}<u(0)<\rho_{2}, \\
1 & \text { if } u(0) \geqslant \rho_{2},\end{cases} \\
\left.w(t):=\left\{\begin{array}{ll}
w(0) & \text { if } X_{t}=\emptyset, \\
-1 & \text { if } X_{t} \neq \emptyset \text { and } u\left(\max X_{t}\right)=\rho_{1}, \\
1 & \text { if } X_{t} \neq \emptyset \text { and } u\left(\max X_{t}\right)=\rho_{2},
\end{array} \quad \forall t \in\right] 0, T\right] .
\end{gathered}
$$


Any function $u \in C^{0}([0, T])$ is uniformly continuous, hence it can only oscillate a finite number of times between $\rho_{1}$ and $\rho_{2}$, if any. Therefore $w$ can just have a finite number of jumps between -1 and 1, if any; hence the total variation of $w$ in $[0, T]$ is finite, i.e., $w \in B V(0, T)$.

Completion. The relay operator is not closed in natural function spaces, because of its discontinuity, cf. [10, Chap. VI]. A suitable extension of this operator is then needed, in view of coupling with PDEs. Following [10], we introduce the (multi-valued) completed relay operator, $k_{\rho}$. For any $u \in C^{0}([0, T])$ and any $\xi \in[-1,1]$, we set $w \in k_{\rho}(u, \xi)$ if and only if $w$ is measurable in $] 0, T$,

$$
w(0):= \begin{cases}-1 & \text { if } u(0)<\rho_{1}, \\ \xi & \text { if } \rho_{1} \leqslant u(0) \leqslant \rho_{2} \\ 1 & \text { if } u(0)>\rho_{2},\end{cases}
$$

and, for any $t \in] 0, T]$,

$$
\begin{gathered}
w(t) \in \begin{cases}\{-1\} & \text { if } u(t)<\rho_{1}, \\
{[-1,1]} & \text { if } \rho_{1} \leqslant u(t) \leqslant \rho_{2}, \\
\{1\} & \text { if } u(t)>\rho_{2},\end{cases} \\
\begin{cases}\text { if } u(t) \neq \rho_{1}, \rho_{2}, & \text { then } w \text { is constant in a neighbourhood of } t, \\
\text { if } u(t)=\rho_{1}, & \text { then w is nonincreasing in a neighbourhood of } t, \\
\text { if } u(t)=\rho_{2}, & \text { then w is nondecreasing in a neighbourhood of } t .\end{cases}
\end{gathered}
$$

The graph of $k_{\rho}$ in the $(u, w)$-plane invades the whole rectangle $\left[\rho_{1}, \rho_{2}\right] \times[-1,1]$, cf. Fig. 3. Moreover, $w \in B V(0, T)$ for any $u \in C^{0}([0, T])$, by the argument we used for $h_{\rho}$.

The operator $k_{\rho}$ is the closure of $h_{\rho}$ in certain function spaces which are relevant for the analysis of PDEs, cf. [10, Chap. VI]. The definition of $k_{\rho}$ can also be justified by means of the Preisach model; see below at the end of this section.

Reformulation of the relay operator. In view of the analysis of PDEs, it is convenient to provide an alternative formulation of the completed relay operator. It is easy to see that (3.4) is equivalent to the system

$$
\left\{\begin{array}{l}
(w-1)\left(u-\rho_{2}\right) \geqslant 0, \\
\left.(w+1)\left(u-\rho_{1}\right) \geqslant 0, \quad \text { a.e. in }\right] 0, T[. \\
|w| \leqslant 1,
\end{array}\right.
$$




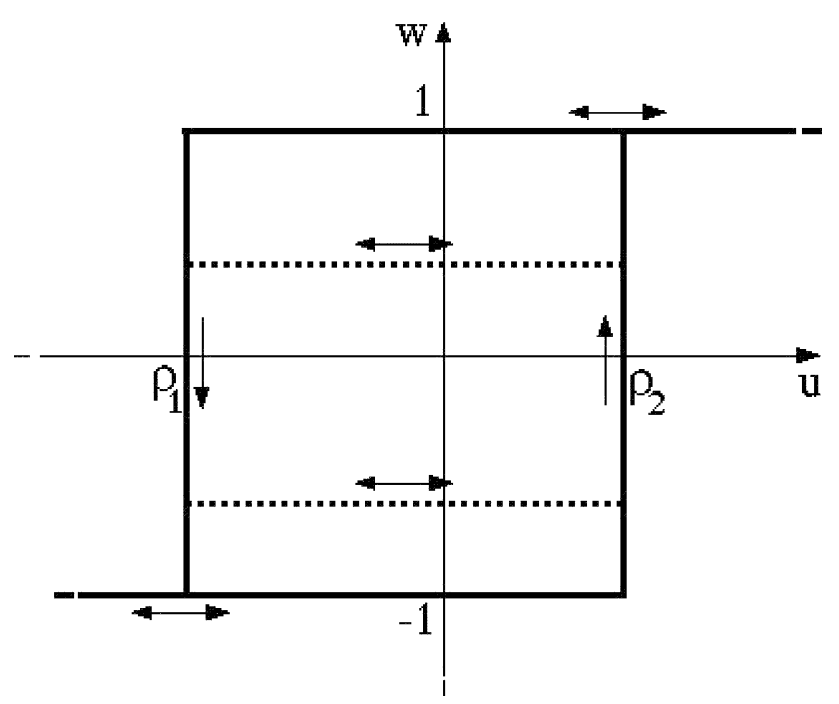

Fig. 3. Completed relay operator. Here the pair $(u, w)$ can also attain any value of the rectangle $\left[\rho_{1}, \rho_{2}\right] \times[-1,1]$.

If $w \in W^{1,1}(0, T)$, the dynamics (3.5) is tantamount to

$$
\left.\left.\int_{0}^{t} \frac{\mathrm{d} w}{\mathrm{~d} \tau} u \mathrm{~d} \tau=\int_{0}^{t}\left[\left(\frac{\mathrm{d} w}{\mathrm{~d} \tau}\right)^{+} \rho_{2}-\left(\frac{\mathrm{d} w}{\mathrm{~d} \tau}\right)^{-} \rho_{1}\right] \mathrm{d} \tau=: \Psi_{\rho}(w, t) \quad \forall t \in\right] 0, T\right] .
$$

In continuum dynamics and in electromagnetism a quantity like $\Psi_{\rho}(w, t)$ represents the dissipated energy.

If the pair $(u, w)$ moves along a closed loop in $[0, t]$, then $\Psi_{\rho}(w, t)$ equals the area of the region bounded by the loop. The condition (3.7) is extended to $w \in B V(0, T)$, provided that we set

$$
\left.\left.\Psi_{\rho}(w, t):=\int_{0}^{t} \rho_{2} \mathrm{~d} w^{+}-\int_{0}^{t} \rho_{1} \mathrm{~d} w^{-} \quad \forall t \in\right] 0, T\right]
$$

and interpret the latter expression as a Stieltjes integral. Notice that

$$
\begin{aligned}
\Psi_{\rho}(w, t) & =\int_{0}^{t} \rho_{2} \mathrm{~d} w^{+}-\int_{0}^{t} \rho_{1} \mathrm{~d} w^{-}=\frac{\rho_{2}+\rho_{1}}{2} \int_{0}^{t} \mathrm{~d} w+\frac{\rho_{2}-\rho_{1}}{2} \int_{0}^{t}|\mathrm{~d} w| \\
& \left.\left.=\frac{\rho_{2}+\rho_{1}}{2}[w(t)-w(0)]+\frac{\rho_{2}-\rho_{1}}{2} \int_{0}^{t}|\mathrm{~d} w| \quad \forall t \in\right] 0, T\right], \forall w \in B V(0, T),
\end{aligned}
$$

and that the total variation, $\int_{0}^{t}|\mathrm{~d} w|$, equals the total mass of the Borel measure $\mathrm{d} w / \mathrm{d} \tau$ in $C^{0}([0, t])^{\prime}$; i.e., $\int_{0}^{t}|\mathrm{~d} w|=\|\mathrm{d} w / \mathrm{d} \tau\|_{C^{0}([0, t])^{\prime}}$. 
The condition (3.4) entails that $\int_{0}^{t} u \mathrm{~d} w \leqslant \Psi_{\rho}(w, t)$, independently from the dynamics; (3.7) is then equivalent to the opposite inequality. Therefore the system (3.4) and (3.5) is equivalent to the confinement condition (3.6) coupled with the dissipation inequality

$$
\left.\left.\int_{0}^{t} u \mathrm{~d} w \geqslant \Psi_{\rho}(w, t) \quad \forall t \in\right] 0, T\right] .
$$

Whenever $u \in C^{0}([0, T]) \cap B V(0, T)$, (3.9) also reads

$$
\left.\left.\int_{0}^{t} u \mathrm{~d}(u+w)-\frac{1}{2}\left[u(t)^{2}-u(0)^{2}\right] \geqslant \Psi_{\rho}(w, t) \quad \forall t \in\right] 0, T\right] .
$$

Notice that (3.9) is also equivalent to the variational inequality

$$
\left.\left.\int_{0}^{t}(u-v) \mathrm{d} w \geqslant 0 \quad \forall v \in\left[\rho_{1}, \rho_{2}\right], \forall t \in\right] 0, T\right] .
$$

The above formulation of the relay operator can be extended to space-distributed systems, just assuming that $u(x, \cdot) \in C^{0}([0, T]), w(x, \cdot) \in B V(0, T)$, and (3.3), (3.6) and (3.9) hold a.e. in $\Omega$. Let us set $\left.Q_{t}:=\Omega \times\right] 0, t$ [ for any $t>0$. In alternative, (3.9) may also be extended by requiring that

$$
\begin{aligned}
& C^{0}(\bar{Q})\left\langle u, \frac{\partial w}{\partial \tau}\right\rangle_{C^{0}\left(\bar{Q}_{t}\right)^{\prime}} \\
& \quad \geqslant \frac{\rho_{2}+\rho_{1}}{2} \iint_{Q_{t}}\left[w(x, \tau)-w^{0}(x)\right] \mathrm{d} x \mathrm{~d} \tau+\frac{\rho_{2}-\rho_{1}}{2}\left\|\frac{\partial w}{\partial \tau}\right\|_{C^{0}\left(\bar{Q}_{t}\right)^{\prime}} \\
& \left.\left.\quad=: \int_{\bar{\Omega}} \Psi_{\rho}(w, t) \quad \forall t \in\right] 0, T\right] .
\end{aligned}
$$

Here we denote the total mass of the Borel measure $\Psi_{\rho}(w, t)$ in $\bar{\Omega}$ by $\int_{\bar{\Omega}} \Psi_{\rho}(w, t)$ (without the $\mathrm{d} x$ ), and reserve the notation $\int_{\Omega} \Psi_{\rho}(w, t) \mathrm{d} x$ (with the $\mathrm{d} x$ ) for the case in which $\Psi_{\rho}(w(x, \cdot), t)$ is Lebesgue integrable.

Preisach integral. Let $\mu$ be a finite Borel measure on the half-plane $\mathcal{P}:=\{\rho:=$ $\left.\left(\rho_{1}, \rho_{2}\right) \in \mathbf{R}^{2}: \rho_{1}<\rho_{2}\right\}$, and define the completed Preisach operator $\mathcal{K}_{\mu}:=\int_{\mathcal{P}} k_{\rho} \mathrm{d} \mu(\rho)$.

More precisely, for any $u \in C^{0}([0, T])$ and any measurable function $\xi: \mathcal{P} \rightarrow\{-1,1\}$, we set $\tilde{w} \in \mathcal{K}_{\mu}(u, \xi)$ iff there exists a measurable function $\left.w: \mathcal{P} \times\right] 0, T[\rightarrow[-1,1]$ such that $w_{\rho} \in k_{\rho}\left(u, \xi_{\rho}\right)$ a.e. in $\left.\mathcal{P} \times\right] 0, T$ [ (w.r.t. the product of the Preisach and Lebesgue measures), and $\tilde{w}=\int_{\mathcal{P}} w_{\rho} \mathrm{d} \mu(\rho)$ a.e. in $] 0, T$. For any $\rho \in \mathcal{P}$ the inclusion $w_{\rho} \in k_{\rho}\left(u, \xi_{\rho}\right)$ can be represented by (3.3), (3.6) and (3.9).

If the measure $\mu$ has no masses concentrated either in points or on lines parallel to any coordinate axis, then $\mathcal{K}_{\mu}$ operates in $C^{0}([0, T])$ and is continuous with respect to the uniform topology. Under further hypotheses on $\mu, \mathcal{K}_{\mu}$ operates in smaller spaces, or 
is invertible, or fulfils other properties; for instance, if $\mu \geqslant 0$ then any hysteresis branch is nondecreasing. For these and other properties of the Preisach model see, e.g., [10, Chap. IV]. Notice that $\mathcal{K}_{\mu}=\mathcal{H}_{\mu}$, whenever the measure $\mu$ is such that $\mathcal{H}_{\mu}$ operates in $C^{0}([0, T])$.

In view of latter application, we notice that, whenever $\rho_{2}+\rho_{1}$ is $\mu$-integrable on $\mathcal{P}$, by (3.8) we have

$$
\begin{aligned}
\int_{A} \Psi_{\rho}\left(w_{\rho}, t\right) \mathrm{d} \mu(\rho)= & \int_{A} \frac{\rho_{2}+\rho_{1}}{2}\left[w_{\rho}(t)-w_{\rho}(0)\right] \mathrm{d} \mu(\rho) \\
& +\int_{A} \frac{\rho_{2}-\rho_{1}}{2}\left(\int_{0}^{t}\left|\mathrm{~d} w_{\rho}\right|\right) \mathrm{d} \mu(\rho) \\
& \forall t \in] 0, T], \forall \text { measurable set } A \subset \mathcal{P} .
\end{aligned}
$$

Finally, we briefly outline how the definition of the completed relay operator, $k_{\rho}$, can be justified by means of the Preisach model. According to this model each relay $h_{\rho}$ corresponds to a Dirac measure, $\mu$, concentrated at the point $\rho \in \mathcal{P}$. In order to approximate the relay, it is then natural to smear out that measure by a sequence of absolutely continuous measures, $\left\{\mu_{n}\right\}$, having a bell-shaped density w.r.t. to the Lebesgue measure. It is not difficult to show that, as $n \rightarrow \infty$, the Preisach operators corresponding to the approximating measures converge to the completed relay operator, $k_{\rho}$, pointwise in $C^{0}([0, T])$.

\section{Weak formulation of the PDE for the relay operator}

We shall deal with Eq. (1.1) for $A:=-\Delta$ (associated with the homogeneous Dirichlet condition). We assume that

$$
\begin{aligned}
& u^{0}, w^{0} \in L^{2}(\Omega), \quad F \in L^{2}\left(0, T ; H^{-1}(\Omega)\right), \\
& \left|w^{0}\right| \leqslant 1, \quad w^{0}=-1 \text { if } u^{0}<\rho_{1}, \quad w^{0}=1 \text { if } u^{0}>\rho_{2} \quad \text { a.e. in } \Omega,
\end{aligned}
$$

and provide a weak formulation of the initial- and boundary-value problem associated with Eq. (1.4).

We denote the duality pairing between $H^{-1}(\Omega)$ and $H_{0}^{1}(\Omega)$ by $\langle\cdot, \cdot\rangle$, and the trace operator by $\gamma_{0}$.

Problem 1. - To find $U \in H^{1}(Q)$ and $w \in L^{\infty}(Q)$ such that $\gamma_{0} U=0$ a.e. in $(\Omega \times\{0\}) \cup(\partial \Omega \times] 0, T[),|w| \leqslant 1$ a.e. in $Q$, and $\partial w / \partial t \in C^{0}(\bar{Q})^{\prime}$. Moreover, we set

$$
u:=\frac{\partial U}{\partial t} \quad \text { a.e. in } Q, \quad w(\cdot, 0):=w^{0} \quad \text { a.e. in } \Omega,
$$

and require that

$$
\iint_{Q}\left[\left(u^{0}+w^{0}-u-w\right) \frac{\partial \eta}{\partial t}+\nabla U \cdot \nabla \eta\right] \mathrm{d} x \mathrm{~d} t=\int_{0}^{T}\langle F, \eta\rangle \mathrm{d} t
$$




$$
\begin{gathered}
\forall \eta \in C^{1}(\bar{Q}), \eta=0 \text { a.e. in }(\Omega \times\{T\}) \cup(\partial \Omega \times] 0, T[), \\
(w-1)\left(u-\rho_{2}\right) \geqslant 0, \quad(w+1)\left(u-\rho_{1}\right) \geqslant 0 \quad \text { a.e. in } Q, \\
\frac{1}{2} \int_{\Omega}\left[u(x, t)^{2}+|\nabla U(x, t)|^{2}-u^{0}(x)^{2}\right] \mathrm{d} x+\int_{\bar{\Omega}} \Psi_{\rho}(w, t) \leqslant \int_{0}^{t}\langle F, u\rangle \mathrm{d} \tau \\
\text { for a.a. } t \in] 0, T[.
\end{gathered}
$$

See (3.12) for the definition of $\int_{\bar{\Omega}} \Psi_{\rho}(w, t)$.

The initial condition in (4.2) is meaningful, on account of the regularity of $w$.

Interpretation. (4.3) is equivalent to the system

$$
\begin{array}{ll}
\frac{\partial}{\partial t}(u+w)-\Delta U=F & \text { in } H^{-1}(Q), \\
\left.(u+w)\right|_{t=0}=u^{0}+w^{0} & \text { in } H^{-1}(\Omega) .
\end{array}
$$

Differentiating (4.6) in time, we get

$$
\frac{\partial^{2}}{\partial t^{2}}(u+w)-\Delta u=f \quad \text { in } \mathcal{D}^{\prime}(Q) .
$$

A comparison of the terms of (4.6) yields $\frac{\partial}{\partial t}(u+w) \in L^{2}\left(0, T ; H^{-1}(\Omega)\right)$; the initial condition (4.7) is then meaningful.

For a moment let us assume that $u \in L^{2}\left(0, T ; H_{0}^{1}(\Omega)\right)$. Multiplying (4.6) by $u$ and integrating in time, we get

$$
\left.\int_{0}^{t}\left\langle\frac{\partial}{\partial \tau}(u+w)-F, u\right\rangle \mathrm{d} \tau+\frac{1}{2} \int_{\Omega}|\nabla U(x, t)|^{2} \mathrm{~d} x=0 \quad \text { for a.a. } t \in\right] 0, T[
$$

(4.5) is then equivalent to

$$
\begin{aligned}
& \int_{0}^{t}\left\langle\frac{\partial}{\partial \tau}(u+w), u\right\rangle \mathrm{d} \tau-\frac{1}{2} \int_{\Omega}\left[u(x, t)^{2}-u^{0}(x)^{2}\right] \mathrm{d} x \geqslant \int_{\bar{\Omega}} \Psi_{\rho}(w(x, \cdot), t) \\
& \quad \text { for a.a. } t \in] 0, T[.
\end{aligned}
$$

This inequality can be compared with (3.10), which jointly with (3.6) and with the second condition of (4.2) represents the hysteresis relation

$$
w \in k_{\rho}\left(u, w^{0}\right) \quad \text { a.e. in } \Omega .
$$

Therefore we can regard Problem 1 as a weak formulation of an initial- and boundaryvalue problem associated to the system (4.6) and (4.11).

Finally, we notice that, on account of the equivalence between (3.9) and (3.11), in Problem 1 (4.5) might be replaced by the following variational inequality, for any $v \in H^{1}(Q)$ such that $\rho_{1} \leqslant v \leqslant \rho_{2}$ a.e. in $Q$, 


$$
\begin{aligned}
& \frac{1}{2} \int_{\Omega}\left[u(x, t)^{2}+|\nabla U(x, t)|^{2}-u^{0}(x)^{2}\right] \mathrm{d} x+\int_{\Omega}\left[u(x, t) v(x, t)-u^{0}(x) v(x, 0)\right] \mathrm{d} x \\
& \left.\quad+\int_{0}^{t} \mathrm{~d} \tau \int_{\Omega}\left[u \frac{\partial v}{\partial \tau}+\nabla U \cdot \nabla v\right] \mathrm{d} x \leqslant \int_{0}^{t}\langle F, u-v\rangle \mathrm{d} \tau \quad \text { for a.a. } t \in\right] 0, T[, \quad
\end{aligned}
$$

which is formally equivalent to

$$
\left.\int_{0}^{t} \mathrm{~d} \tau \int_{\Omega}\left[\frac{\partial u}{\partial \tau}(u-v)+\nabla U \cdot \nabla(u-v)\right] \mathrm{d} x \leqslant \int_{0}^{t}\langle F, u-v\rangle \mathrm{d} \tau \quad \text { for a.a. } t \in\right] 0, T[.
$$

\section{Existence result for the relay operator}

At first we recall a result of interpolation of spaces of vector-valued functions.

Lemma 5.1 (see, e.g., Triebel [9, p. 128]). - Let $A_{0}$ and $A_{1}$ be Banach spaces, subspaces of the same separated topological vector space. Let

$$
1 \leqslant p_{0}, p_{1}<+\infty, \quad 0<\theta<1, \quad \frac{1}{p}=\theta \frac{1}{p_{0}}+(1-\theta) \frac{1}{p_{1}} .
$$

Denoting by $\left(A_{0}, A_{1}\right)_{[\theta]}$ the complex interpolation space, we then have

$$
\left(L^{p_{0}}\left(\Omega ; A_{0}\right), L^{p_{1}}\left(\Omega ; A_{1}\right)\right)_{[\theta]}=L^{p}\left(\Omega ;\left(A_{0}, A_{1}\right)_{[\theta]}\right) .
$$

This statement allows us to derive the following compensated compactness result.

LeMmA 5.2. - Let $z, w$ and the sequences $\left\{z_{m}\right\},\left\{w_{m}\right\}$ be such that

$$
\begin{aligned}
& z_{m} \rightarrow z \quad \text { weakly in } L^{2}(Q) \cap H^{-1}\left(0, T ; H^{1}(\Omega)\right), \\
& w_{m} \rightarrow w \text { weakly star in } L^{\infty}(Q), \\
& \left\|w_{m}\right\|_{L^{1}(\Omega ; B V(0, T))} \leqslant \text { Constant. }
\end{aligned}
$$

Then

$$
\iint_{Q} w_{m} z_{m} \mathrm{~d} x \mathrm{~d} t \rightarrow \iint_{Q} w z \mathrm{~d} x \mathrm{~d} t
$$

Proof. - Let $\theta, p, r$ be such that

$$
0<\theta<1, \quad 2<p<+\infty, \quad 0<r<\frac{1}{p}, \quad \frac{\theta}{p}+1-\theta=\frac{1}{2} .
$$

Obviously these conditions are nonempty. By Sobolev inclusion and by interpolation, we have

$$
B V(0, T) \subset H^{r}(0, T), \quad\left(L^{2}(0, T), H^{r}(0, T)\right)_{[\theta]}=H^{r(1-\theta)}(0, T) .
$$

Lemma 5.1 then yields 


$$
\begin{aligned}
L^{\infty}(Q) \cap L^{1}(\Omega ; B V(0, T)) & \subset L^{\infty}(Q) \cap L^{1}\left(\Omega ; H^{r}(0, T)\right) \\
& \subset\left(L^{p}\left(\Omega ; L^{2}(0, T)\right), L^{1}\left(\Omega ; H^{r}(0, T)\right)\right)_{[\theta]} \\
& =L^{2}\left(\Omega ;\left(L^{2}(0, T), H^{r}(0, T)\right)_{[\theta]}\right)=L^{2}\left(\Omega ; H^{r(1-\theta)}(0, T)\right) .
\end{aligned}
$$

Note that $0<r(1-\theta)<\frac{1}{p}<\frac{1}{2}$. Again by interpolation, see, e.g., Lions and Magenes [7, Chap. IV], we also have

$$
\begin{aligned}
L^{2}(Q) \cap H^{-1}\left(0, T ; H^{1}(\Omega)\right) & =L^{2}\left(\Omega ; L^{2}(0, T)\right) \cap H^{1}\left(\Omega ; H^{-1}(0, T)\right) \\
& \subset L^{2}\left(\Omega ; H^{-r(1-\theta)}(0, T)\right)=L^{2}\left(\Omega ; H^{r(1-\theta)}(0, T)\right)^{\prime}
\end{aligned}
$$

with compact injection. Therefore (5.4) holds.

THEOREM 5.3 (Existence). - If (4.1) is fulfilled and $F \in W^{1,1}\left(0, T ; H^{-1}(\Omega)\right)$, then there exists a solution $(U, w)$ of Problem 1 such that

$$
U \in W^{1, \infty}\left(0, T ; L^{2}(\Omega)\right) \cap L^{\infty}\left(0, T ; H_{0}^{1}(\Omega)\right) .
$$

Proof. - (i) Approximation. Let us fix any $m \in \mathbf{N}$, set $k:=T / m, u_{m}^{0}:=u^{0}, w_{m}^{0}:=w^{0}$, $F_{1 m}^{n}:=\frac{1}{k} \int_{(n-1) k}^{n k} F_{1}(\cdot, t) \mathrm{d} t$ a.e. in $\Omega, F_{2 m}^{n}:=F_{2}(n k), F_{m}^{n}:=F_{1 m}^{n}+F_{2 m}^{n}$ for $n=1, \ldots, m$, and

$$
G_{\rho}(v, \xi):= \begin{cases}\{-1\} & \text { if } v<\rho_{1}, \\ {[-1, \xi]} & \text { if } v=\rho_{1}, \\ \{\xi\} & \text { if } \rho_{1}<v<\rho_{2}, \quad \forall(v, \xi) \in \mathbf{R} \times[-1,1], \text { cf. Fig. } 4 . \\ {[\xi, 1]} & \text { if } v=\rho_{2}, \\ \{1\} & \text { if } v>\rho_{2},\end{cases}
$$

We are now able to introduce a time-discretization scheme of implicit type for our problem.

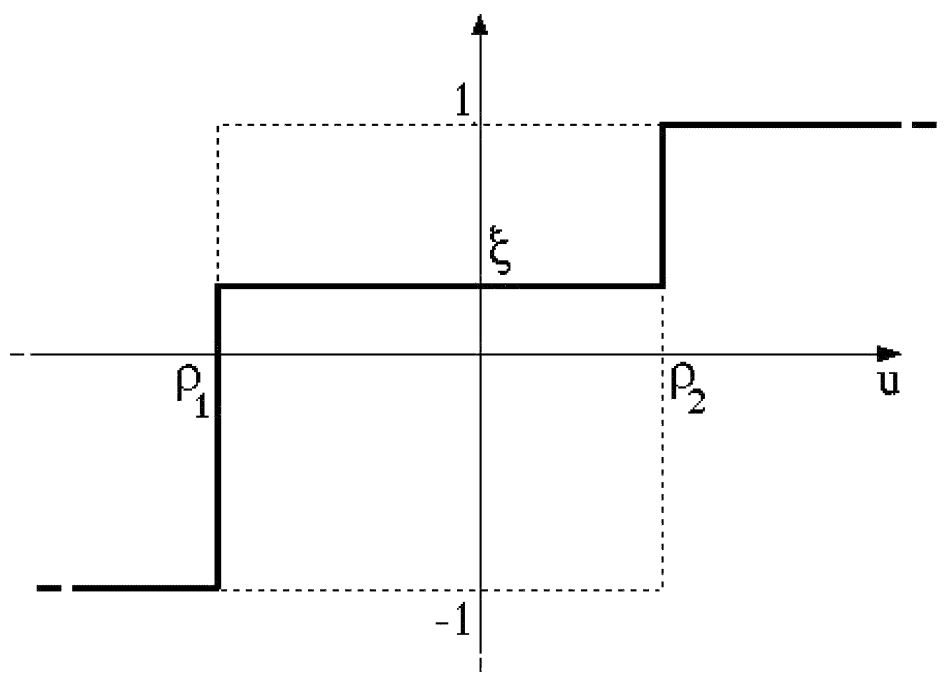

Fig. 4. Graph of the multi-valued function $G_{\rho}(\cdot, \xi)$, for a fixed $\xi \in[-1,1]$. 
Problem $\mathbf{1}_{m}$. - To find $u_{m}^{n} \in H_{0}^{1}(\Omega)$ and $w_{m}^{n} \in L^{\infty}(\Omega)$ for $n=1, \ldots, m$, such that

$$
\begin{gathered}
w_{m}^{n} \in G_{\rho}\left(u_{m}^{n}, w_{m}^{n-1}\right) \quad \text { a.e. in } \Omega, \text { for } n=1, \ldots, m, \\
\frac{u_{m}^{n}-u_{m}^{n-1}}{k}+\frac{w_{m}^{n}-w_{m}^{n-1}}{k}-k \sum_{j=1}^{n} \Delta u_{m}^{j}=F_{m}^{n} \quad \text { in } H^{-1}(\Omega), \text { for } n=1, \ldots, m .
\end{gathered}
$$

For any $n$ and for a.a. $x \in \Omega$, the maximal monotone function $G_{\rho}\left(\cdot, w_{m}^{n-1}(x)\right)$ is the subdifferential of a lower semicontinuous and convex function, $g_{\rho}(\cdot, x)$, which depends measurably on $x$. The functional

$$
\begin{aligned}
& J_{m}^{n}: v \mapsto \\
& \int_{\Omega}\left[\frac{v^{2}}{2}+g_{\rho}(v(x), x)+\frac{k^{2}}{2}|\nabla v|^{2}+k^{2} \sum_{j=1}^{n-1} \nabla u_{m}^{n-1} \cdot \nabla v-\left(u_{m}^{n-1}+w_{m}^{n-1}\right) v\right] \mathrm{d} x \\
& \quad-k\left\langle F_{m}^{n}, v\right\rangle
\end{aligned}
$$

is (strictly) convex, lower semicontinuous and coercive on $H_{0}^{1}(\Omega)$. Hence it has a (unique) minimizer $u_{m}^{n}$, and $\partial J_{m}^{n}\left(u_{m}^{n}\right) \ni 0$ in $H^{-1}(\Omega)$. This inclusion is equivalent to the system (5.7) and (5.8).

(ii) A priori estimates. Preliminarly, for any family $\left\{v_{m}^{n}\right\}_{n=1, \ldots, m}$ of functions $\Omega \rightarrow \mathbf{R}$ let us denote by $v_{m}$ the time interpolate of $v_{m}^{0}:=v^{0}, v_{m}^{1}, \ldots, v_{m}^{m}$ a.e. in $\Omega$, and set $\bar{v}_{m}(\cdot, t):=v_{m}^{n}$ a.e. in $\Omega$, if $(n-1) k<t \leqslant n k$, for $n=1, \ldots, m$.

Let us multiply Eq. (5.8) by $k u_{m}^{n}$, and sum for $n=0, \ldots, \ell$, for any $\ell \in\{1, \ldots, m\}$. By (5.7) we have

$$
\begin{aligned}
& \sum_{n=1}^{\ell}\left(w_{m}^{n}-w_{m}^{n-1}\right) u_{m}^{n} \geqslant \sum_{n=1}^{\ell}\left[\left(w_{m}^{n}-w_{m}^{n-1}\right)^{+} \rho_{2}-\left(w_{m}^{n}-w_{m}^{n-1}\right)^{-} \rho_{1}\right]=\Psi_{\rho}\left(w_{m}, \ell k\right) \\
& \quad \text { a.e. in } \Omega \text {, for } \ell=1, \ldots, m .
\end{aligned}
$$

Setting $U_{m}^{n}:=k \sum_{j=1}^{n} u_{m}^{j}$ a.e. in $\Omega$ for $n=1, \ldots, m$, we then get

$$
\begin{aligned}
& \frac{1}{2} \int_{\Omega}\left[\left(u_{m}^{\ell}\right)^{2}+\left|\nabla U_{m}^{\ell}\right|^{2}-\left(u^{0}\right)^{2}\right] \mathrm{d} x+\int_{\Omega} \Psi_{\rho}\left(w_{m}, \ell k\right) \mathrm{d} x \leqslant k \sum_{n=1}^{\ell}\left\langle F_{m}^{n}, u_{m}^{n}\right\rangle \\
& \quad \text { for } \ell=1, \ldots, m .
\end{aligned}
$$

A standard calculation then yields

$$
\left\|U_{m}\right\|_{W^{1, \infty}\left(0, T ; L^{2}(\Omega)\right) \cap L^{\infty}\left(0, T ; H_{0}^{1}(\Omega)\right)},\left\|\Psi_{\rho}\left(w_{m}, \cdot\right)\right\|_{L^{\infty}\left(0, T ; L^{1}(\Omega)\right)} \leqslant C_{1} .
$$

(Throughout this paper by $C_{1}, C_{2}, \ldots$ we denote suitable positive constants independent of $m$.) Hence by (3.8) we get

$$
\left\|w_{m}\right\|_{L^{1}(\Omega ; B V(0, T))} \leqslant C_{2}
$$


(iii) Limit procedure. By the above estimates, there exist $U, w$ such that, as $m \rightarrow \infty$ along a suitable sequence,

$$
\begin{aligned}
U_{m} \rightarrow U \quad \text { weakly star in } W^{1, \infty}\left(0, T ; L^{2}(\Omega)\right) \cap L^{\infty}\left(0, T ; H_{0}^{1}(\Omega)\right), \\
w_{m} \rightarrow w \quad \text { weakly star in } L^{\infty}(Q), \\
\frac{\partial w_{m}}{\partial t} \rightarrow \frac{\partial w}{\partial t} \quad \text { weakly star in } C^{0}(\bar{Q})^{\prime} .
\end{aligned}
$$

Setting $U_{m}:=\int_{0}^{t} \bar{u}_{m}(\cdot, \tau) \mathrm{d} \tau$ a.e. in $Q,(5.8)$ reads

$$
\left.\frac{\partial}{\partial t}\left(u_{m}+w_{m}\right)-\Delta U_{m}=\bar{F}_{m} \quad \text { in } H^{-1}(\Omega), \text { a.e. in }\right] 0, T[,
$$

and passing to the limit we get (4.6), whence (4.3) follows on account of the initial condition (4.7). (5.7) entails

$$
\left(\bar{w}_{m}-1\right)\left(\bar{u}_{m}-\rho_{2}\right) \geqslant 0, \quad\left(\bar{w}_{m}+1\right)\left(\bar{u}_{m}-\rho_{1}\right) \geqslant 0 \quad \text { a.e. in } Q,
$$

whence, for any nonnegative $\varphi \in \mathcal{D}(Q)$,

$$
\iint_{Q}\left(\bar{w}_{m}-1\right)\left(\bar{u}_{m}-\rho_{2}\right) \varphi(x, t) \mathrm{d} x \mathrm{~d} t \geqslant 0, \quad \iint_{Q}\left(\bar{w}_{m}+1\right)\left(\bar{u}_{m}-\rho_{1}\right) \varphi(x, t) \mathrm{d} x \mathrm{~d} t \geqslant 0 .
$$

Applying Lemma 5.1 to the sequences $\left\{z_{m}\right\}:=\left\{\bar{u}_{m} \varphi\right\}$ and $\left\{w_{m}\right\}$, we have

$$
\iint_{Q} w_{m} \bar{u}_{m} \varphi \mathrm{d} x \mathrm{~d} t \rightarrow \iint_{Q} w u \varphi \mathrm{d} x \mathrm{~d} t
$$

Hence

$$
\iint_{Q} \bar{w}_{m} \bar{u}_{m} \varphi \mathrm{d} x \mathrm{~d} t \rightarrow \iint_{Q} w u \varphi \mathrm{d} x \mathrm{~d} t
$$

as $\left\|\bar{w}_{m}-w_{m}\right\|_{L^{2}(Q)} \rightarrow 0$. Passing to the limit in (5.19) we then get

$$
\iint_{Q}(w-1)\left(u-\rho_{2}\right) \varphi(x, t) \mathrm{d} x \mathrm{~d} t \geqslant 0, \quad \iint_{Q}(w+1)\left(u-\rho_{1}\right) \varphi(x, t) \mathrm{d} x \mathrm{~d} t \geqslant 0,
$$

for any nonnegative $\varphi \in \mathcal{D}(Q)$; this is equivalent to (4.4). (5.11) yields

$$
\begin{aligned}
& \frac{1}{2} \int_{\Omega}\left[\bar{u}_{m}^{2}+\left|\nabla \bar{U}_{m}\right|^{2}-\left(u^{0}\right)^{2}\right] \mathrm{d} x+\int_{\Omega} \Psi_{\rho}\left(w_{m}, t\right) \mathrm{d} x \leqslant \int_{0}^{t}\left\langle\bar{F}_{m}, \bar{u}_{m}\right\rangle \mathrm{d} \tau \\
& \quad \text { for a.a. } t \in] 0, T[.
\end{aligned}
$$

Integrating in ]0, $T$ [ and passing to the inferior limit as $m \rightarrow \infty$, by lower semicontinuity we finally get an inequality equivalent to (4.5). 


\section{Weak formulation of the PDE for the Preisach operator}

We assume that

$\mu$ is a finite positive Borel measure on $\mathcal{P}:=\left\{\rho:=\left(\rho_{1}, \rho_{2}\right) \in \mathbf{R}^{2}: \rho_{1}<\rho_{2}\right\}$,

and equip $\Omega \times \mathcal{P}$ and $Q \times \mathcal{P}$ with the product of the Lebesgue and Preisach measures, $\mathcal{L}^{N} \times \mu\left(\mathcal{L}^{(N+1)} \times \mu\right.$, respectively). We also set

$$
\begin{aligned}
\iint_{\bar{\Omega} \times \mathcal{P}} \Psi_{\rho}\left(w_{\rho}, t\right):= & \int_{\mathcal{P}} \mathrm{d} \mu(\rho) \frac{\rho_{2}+\rho_{1}}{2} \iint_{Q_{t}}\left[w_{\rho}(x, \tau)-w_{\rho}^{0}(x)\right] \mathrm{d} x \mathrm{~d} \tau \\
& \left.\left.+\left\|\frac{\rho_{2}-\rho_{1}}{2} \frac{\partial w_{\rho}}{\partial \tau}\right\|_{C^{0}\left(\bar{Q}_{t} \times \mathcal{P}\right)^{\prime}} \quad \forall t \in\right] 0, T\right],
\end{aligned}
$$

and reserve the notation $\iint_{\Omega \times \mathcal{P}} \Psi_{\rho}\left(w_{\rho}, t\right) \mathrm{d} x \mathrm{~d} \mu(\rho)$ for the case in which $\Psi_{\rho}\left(w_{\rho}(x, \cdot), t\right)$ is Lebesgue integrable w.r.t. $\mathcal{L}^{N} \times \mu$. We assume that

$$
\begin{aligned}
& u^{0} \in L^{2}(\Omega), \quad F \in L^{2}\left(0, T ; H^{-1}(\Omega)\right), \quad w^{0} \in L^{\infty}(\Omega \times \mathcal{P}), \\
& \left|w_{\rho}^{0}\right| \leqslant 1, \quad w_{\rho}^{0}=-1 \text { if } u^{0}<\rho_{1}, \quad w_{\rho}^{0}=1 \text { if } u^{0}>\rho_{2} \quad \text { a.e. in } \Omega \times \mathcal{P},
\end{aligned}
$$

and provide a weak formulation of the initial- and boundary-value problem associated with (1.4), for $\mathcal{F}$ equal to the Preisach operator.

Problem 2. - To find $U \in H^{1}(Q)$ and $w \in L^{\infty}(Q \times \mathcal{P})$ such that $\gamma_{0} U=0$ a.e. in $(\Omega \times\{0\}) \cup(\partial \Omega \times] 0, T[),|w| \leqslant 1$ a.e. in $Q \times \mathcal{P}$, and $\partial w / \partial \tau \in C^{0}(\bar{Q} \times \mathcal{P})^{\prime}$. Moreover, we set

$$
\begin{array}{r}
u:=\frac{\partial U}{\partial t} \text { a.e. in } Q, \quad \tilde{w}^{0}:=\int_{\mathcal{P}} w_{\rho}^{0} \mathrm{~d} \mu(\rho), \quad \tilde{w}:=\int_{\mathcal{P}} w_{\rho} \mathrm{d} \mu(\rho) \text { a.e. in } Q, \\
w_{\rho}(\cdot, 0):=w_{\rho}^{0} \quad \text { a.e. in } \Omega \times \mathcal{P}, \quad \tilde{w}^{0}:=\int_{\mathcal{P}} w_{\rho}^{0} \mathrm{~d} \mu(\rho) \quad \text { a.e. in } \Omega,
\end{array}
$$

and require that

$$
\begin{gathered}
\iint_{Q}\left[\left(u^{0}+\tilde{w}^{0}-u-\tilde{w}\right) \frac{\partial \eta}{\partial t}+\nabla U \cdot \nabla \eta\right] \mathrm{d} x \mathrm{~d} t=\int_{0}^{T}\langle F, \eta\rangle \mathrm{d} t \\
\forall \eta \in C^{1}(\bar{Q}), \eta=0 \text { a.e. in }(\Omega \times\{T\}) \cup(\partial \Omega \times] 0, T[), \\
\left(w_{\rho}-1\right)\left(u-\rho_{2}\right) \geqslant 0, \quad\left(w_{\rho}+1\right)\left(u-\rho_{1}\right) \geqslant 0 \quad \text { a.e. in } Q \times \mathcal{P}, \\
\frac{1}{2} \int_{\Omega}\left[u(x, t)^{2}+|\nabla U(x, t)|^{2}-u^{0}(x)^{2}\right] \mathrm{d} x+\iint_{\bar{\Omega} \times \mathcal{P}} \Psi_{\rho}\left(w_{\rho}, t\right) \leqslant \int_{0}^{t}\langle F, u\rangle \mathrm{d} \tau \\
\text { for a.a. } t \in] 0, T[.
\end{gathered}
$$


Interpretation. (6.6) is equivalent to the system

$$
\begin{array}{ll}
\frac{\partial}{\partial t}(u+\tilde{w})-\Delta U=F & \text { in } H^{-1}(Q), \\
\left.(u+\tilde{w})\right|_{t=0}=u^{0}+\tilde{w}^{0} & \text { in } H^{-1}(\Omega) .
\end{array}
$$

Similarly to what we saw in the interpretation of Problem 1 , if $u \in L^{2}\left(0, T ; H_{0}^{1}(\Omega)\right)$ then (6.6) yields (4.8), here with $\tilde{w}$ in place of $w .(6.8)$ is then equivalent to

$$
\begin{aligned}
& \int_{0}^{t}\left\langle\frac{\partial}{\partial \tau}(u+\tilde{w}), u\right\rangle \mathrm{d} \tau \geqslant \frac{1}{2} \int_{\Omega}\left[u(x, t)^{2}-u^{0}(x)^{2}\right] \mathrm{d} x+\iint_{\bar{\Omega} \times \mathcal{P}} \Psi_{\rho}\left(w_{\rho}, t\right) \\
& \quad \text { for a.a. } t \in] 0, T[.
\end{aligned}
$$

This can be regarded as a weak formulation of the inequality (3.10) a.e. in $\Omega \times \mathcal{P}$; the opposite inequality follows from the confinement condition (3.6). (By displaying the $\mathrm{d} x$ in the integral expression, we still distinguish the Lebesgue integral on $\Omega$ from the total mass of a Borel measure, cf. Section 3.) (6.7) and (6.8) then account for the hysteresis relation

$$
\left.\tilde{w} \in \mathcal{K}_{\mu}\left(u, w^{0}\right):=\int_{\mathcal{P}} k_{\rho}\left(u, w_{\rho}^{0}\right) \mathrm{d} \mu(\rho) \quad \text { in }\right] 0, T \text {, a.e. in } \Omega .
$$

Therefore Problem 2 can be regarded as a weak formulation of an initial- and boundary-value problem associated to the system (6.9) and (6.12).

\section{Existence result for the Preisach operator}

We still assume that $\mu$ is a finite positive Borel measure on the Preisach half-plane $\mathcal{P}:=\left\{\rho:=\left(\rho_{1}, \rho_{2}\right) \in \mathbf{R}^{2}: \rho_{1}<\rho_{2}\right\}$. The following extension of Lemma 5.2 can be justified by the same argument.

Lemma 7.1.-. Let A be any measurable subset of $\mathcal{P}$. Assume that $z, w$ and the sequences $\left\{z_{m}\right\},\left\{w_{m}\right\}$ are such that

$$
\begin{aligned}
& z_{m} \rightarrow z \text { weakly in } L^{2}(Q) \cap H^{-1}\left(0, T ; H^{1}(\Omega)\right), \text { uniformly w.r.t. } \rho \text { in } A, \\
& w_{m} \rightarrow w \text { weakly star in } L^{\infty}(Q \times A), \\
& \left\|w_{m}\right\|_{L^{1}(\Omega \times A ; B V(0, T))} \leqslant \text { Constant. }
\end{aligned}
$$

Then

$$
\int_{A} \mathrm{~d} \mu \iint_{Q} w_{m} z_{m} \mathrm{~d} x \mathrm{~d} t \rightarrow \int_{A} \mathrm{~d} \mu \iint_{Q} w z \mathrm{~d} x \mathrm{~d} t .
$$

THEOREM 7.2. - Assume that (6.1) and (6.3) hold, that $F \in W^{1,1}\left(0, T ; H^{-1}(\Omega)\right)$, and that

$$
\rho_{1}+\rho_{2} \in L^{1}(\mathcal{P} ; \mu)
$$

Then there exists a solution $(U, w)$ of Problem 2 such that

$$
U \in W^{1, \infty}\left(0, T ; L^{2}(\Omega)\right) \cap L^{\infty}\left(0, T ; H_{0}^{1}(\Omega)\right) .
$$


Proof. - This argument is partly similar to that of Theorem 5.3.

(i) Approximation. Let us fix any $m \in \mathbf{N}$, and define $k, u_{m}^{0}, w_{m}^{0}, F_{m}^{n}, G_{\rho}$ and $g_{\rho}$ as in Section 5.

PRoblem $\mathbf{2}_{m}$. - To find $u_{m}^{n} \in H_{0}^{1}(\Omega)$ and $w_{m}^{n} \in L^{\infty}(\Omega \times \mathcal{P})$ for $n=1, \ldots, m$, such that, setting $\tilde{w}_{m}^{n}:=\int_{\mathcal{P}}\left(w_{m}^{n}\right)_{\rho} \mathrm{d} \mu(\rho)$ a.e. in $\Omega$,

$$
\begin{gathered}
\left(w_{m}^{n}\right)_{\rho} \in G_{\rho}\left(u_{m}^{n},\left(w_{m}^{n-1}\right)_{\rho}\right) \quad \text { a.e. in } \Omega \times \mathcal{P}, \text { for } n=1, \ldots, m, \\
\frac{u_{m}^{n}-u_{m}^{n-1}}{k}+\frac{\tilde{w}_{m}^{n}-\tilde{w}_{m}^{n-1}}{k}-k \sum_{j=1}^{n} \Delta u_{m}^{j}=F_{m}^{n} \quad \text { in } H^{-1}(\Omega), \text { for } n=1, \ldots, m .
\end{gathered}
$$

For any $n$ the functional

$$
\begin{aligned}
& \hat{J}_{m}^{n}: v \mapsto \\
& \int_{\Omega}\left[\frac{v^{2}}{2}+\int_{\mathcal{P}} g_{\rho}(v, x) \mathrm{d} \mu(\rho)+\frac{k^{2}}{2}|\nabla v|^{2}+k^{2} \sum_{j=1}^{n-1} \nabla u_{m}^{j} \cdot \nabla v-\left(u_{m}^{n-1}+\tilde{w}_{m}^{n-1}\right) v\right] \mathrm{d} x \\
& \quad-k\left\langle F_{m}^{n}, v\right\rangle
\end{aligned}
$$

is (strictly) convex, lower semicontinuous and coercive on $H_{0}^{1}(\Omega)$. Hence it has a (unique) minimizer $u_{m}^{n}$, and $\partial \hat{J}_{m}^{n}\left(u_{m}^{n}\right) \ni 0$ in $H^{-1}(\Omega)$. This inclusion is equivalent to the system (7.5) and (7.6).

(ii) A priori estimates. We shall denote piecewise-linear and piecewise-constant interpolate functions as in Section 5. Let us multiply Eq. (7.6) by $k u_{m}^{n}$, and sum for $n=1, \ldots, \ell$, for any $\ell \in\{1, \ldots, m\}$. Integrating (5.10) in $\mathcal{P}$, we have

$$
\sum_{n=1}^{\ell}\left(\tilde{w}_{m}^{n}-\tilde{w}_{m}^{n-1}\right) u_{m}^{n}=\sum_{n=1}^{\ell} \int_{\mathcal{P}}\left[\left(w_{m}^{n}\right)_{\rho}-\left(w_{m}^{n-1}\right)_{\rho}\right] u_{m}^{n} \mathrm{~d} \mu(\rho) \geqslant \iint_{\mathcal{P}} \Psi_{\rho}\left(\left(w_{m}\right)_{\rho}, \ell k\right) \mathrm{d} \mu(\rho)
$$

a.e. in $\Omega$, for $\ell=1, \ldots, m$.

We then get

$$
\begin{aligned}
& \frac{1}{2} \int_{\Omega}\left[\left(u_{m}^{\ell}\right)^{2}+\left|\nabla U_{m}^{\ell}\right|^{2}-\left(u^{0}\right)^{2}\right] \mathrm{d} x+\iint_{\Omega \times \mathcal{P}} \Psi_{\rho}\left(\left(w_{m}\right)_{\rho}, \ell k\right) \mathrm{d} x \mathrm{~d} \mu(\rho) \leqslant k \sum_{n=1}^{\ell}\left\langle F_{m}^{n}, u_{m}^{n}\right\rangle \\
& \text { a.e. in } \Omega \text {, for } \ell=1, \ldots, m .
\end{aligned}
$$

A standard calculation then yields

$$
\left\|U_{m}\right\|_{W^{1, \infty}\left(0, T ; L^{2}(\Omega)\right) \cap L^{\infty}\left(0, T ; H_{0}^{1}(\Omega)\right)},\left\|\Psi_{\rho}\left(\left(w_{m}\right)_{\rho}, \cdot\right)\right\|_{L^{\infty}\left(0, T ; L^{1}(\Omega \times \mathcal{P})\right)} \leqslant C_{3} .
$$

(iii) Limit procedure. By the above estimates, there exist $U, w$ such that, as $m \rightarrow \infty$ along a suitable sequence,

$$
\begin{gathered}
U_{m} \rightarrow U \quad \text { weakly star in } W^{1, \infty}\left(0, T ; L^{2}(\Omega)\right) \cap L^{\infty}\left(0, T ; H_{0}^{1}(\Omega)\right), \\
w_{m} \rightarrow w \quad \text { weakly star in } L^{\infty}(Q \times \mathcal{P}) .
\end{gathered}
$$


Setting $U_{m}:=\int_{0}^{t} \bar{u}_{m}(\cdot, \tau) \mathrm{d} \tau$ a.e. in $Q,(7.6)$ reads

$$
\left.\frac{\partial}{\partial t}\left(u_{m}+\tilde{w}_{m}\right)-\Delta U_{m}=\bar{F}_{m} \quad \text { in } H^{-1}(\Omega), \text { a.e. in }\right] 0, T[,
$$

and passing to the limit we get (6.9), whence (6.6) follows on account of the initial condition (6.10).

(7.5) entails

$$
\left[\left(\bar{w}_{m}\right)_{\rho}-1\right]\left(\bar{u}_{m}-\rho_{2}\right) \geqslant 0, \quad\left[\left(\bar{w}_{m}\right)_{\rho}+1\right]\left(\bar{u}_{m}-\rho_{1}\right) \geqslant 0 \quad \text { a.e. in } Q \times \mathcal{P},
$$

whence, for any nonnegative $\varphi \in \mathcal{D}(Q \times \mathcal{P})$,

$$
\begin{aligned}
& \int_{\mathcal{P}} \mathrm{d} \mu(\rho) \iint_{Q}\left[\left(\bar{w}_{m}\right)_{\rho}-1\right]\left(\bar{u}_{m}-\rho_{2}\right) \varphi \mathrm{d} x \mathrm{~d} t \geqslant 0, \\
& \int_{\mathcal{P}} \mathrm{d} \mu(\rho) \iint_{Q}\left[\left(\bar{w}_{m}\right)_{\rho}+1\right]\left(\bar{u}_{m}-\rho_{1}\right) \varphi \mathrm{d} x \mathrm{~d} t \geqslant 0 .
\end{aligned}
$$

We claim that (see below for the argument)

$$
\int_{\mathcal{P}} \mathrm{d} \mu(\rho) \iint_{Q}\left(\bar{w}_{m}\right)_{\rho} \bar{u}_{m} \varphi \mathrm{d} x \mathrm{~d} t \rightarrow \int_{\mathcal{P}} \mathrm{d} \mu(\rho) \iint_{Q} w_{\rho} u \varphi \mathrm{d} x \mathrm{~d} t .
$$

Passing to the limit in (7.14), we then get a system of two inequalities equivalent to (6.7). (7.8) yields

$$
\frac{1}{2} \int_{\Omega}\left[\bar{u}_{m}^{2}+\left|\nabla \bar{U}_{m}\right|^{2}-\left(u^{0}\right)^{2}\right] \mathrm{d} x+\iint_{\Omega \times \mathcal{P}} \Psi_{\rho}\left(\left(w_{m}\right)_{\rho}, t\right) \mathrm{d} x \mathrm{~d} \mu(\rho) \leqslant \int_{0}^{t}\left\langle\bar{F}_{m}, \bar{u}_{m}\right\rangle \mathrm{d} \tau
$$

for a.a. $t \in] 0, T[$.

Integrating in ]0, $T$ [ and passing to the inferior limit as $m \rightarrow \infty$, by lower semicontinuity we get an inequality equivalent to (6.8).

(iv) Proof of (7.15). For any $\varepsilon>0$, there exists $\delta>0$ such that, setting $\mathcal{P}_{\delta}:=\{\rho:=$ $\left.\left(\rho_{1}, \rho_{2}\right) \in \mathbf{R}^{2}: \rho_{2}-\rho_{1} \geqslant \delta\right\}$, we have $\mu\left(\mathcal{P} \backslash \mathcal{P}_{\delta}\right) \leqslant \varepsilon$. By (3.13), we have

$$
\begin{aligned}
\int_{\mathcal{P}_{\delta}} \mathrm{d} & \mu(\rho) \iint_{Q}\left|\mathrm{~d}\left(w_{m}\right)_{\rho}\right| \leqslant \frac{2}{\delta} \iint_{\Omega \times \mathcal{P}_{\delta}} \mathrm{d} x \mathrm{~d} \mu(\rho) \int_{0}^{T} \frac{\rho_{2}-\rho_{1}}{2}\left|\mathrm{~d}\left(w_{m}(x, \cdot)\right)_{\rho}\right| \\
= & \frac{2}{\delta} \iint_{\Omega \times \mathcal{P}_{\delta}} \Psi_{\rho}\left(\left(w_{m}\right)_{\rho}, T\right) \mathrm{d} x \mathrm{~d} \mu(\rho) \\
& -\frac{2}{\delta} \iint_{\Omega \times \mathcal{P}_{\delta}} \frac{\rho_{2}+\rho_{1}}{2}\left[\left(w_{m}\right)_{\rho}(x, T)-\left(w_{m}\right)_{\rho}(x, 0)\right] \mathrm{d} x \mathrm{~d} \mu(\rho) \\
\leqslant & \frac{2}{\delta} \iint_{\Omega \times \mathcal{P}} \Psi_{\rho}\left(\left(w_{m}\right)_{\rho}, T\right) \mathrm{d} x \mathrm{~d} \mu(\rho)+\frac{2}{\delta} \iint_{\Omega \times \mathcal{P}}\left|\rho_{2}+\rho_{1}\right| \mathrm{d} x \mathrm{~d} \mu(\rho) .
\end{aligned}
$$


By (7.9), $w_{m}$ is therefore uniformly bounded in $L^{1}\left(\Omega \times \mathcal{P}_{\delta} ; B V(0, T)\right)$. We can then apply Lemma 7.1 to the sequences $\left\{z_{m}\right\}=\left\{\bar{u}_{m} \varphi\right\}$ and $\left\{\bar{w}_{m}\right\}$, which yields

$$
\int_{\mathcal{P}_{\delta}} \mathrm{d} \mu(\rho) \iint_{Q}\left(\bar{w}_{m}\right)_{\rho} \bar{u}_{m} \varphi \mathrm{d} x \mathrm{~d} t \rightarrow \int_{\mathcal{P}_{\delta}} \mathrm{d} \mu(\rho) \iint_{Q} w_{\rho} u \varphi \mathrm{d} x \mathrm{~d} t .
$$

Finally, we have

$$
\begin{aligned}
& \left|\int_{\mathcal{P}} \mathrm{d} \mu(\rho) \iint_{Q}\left(\bar{w}_{m}\right)_{\rho} \bar{u}_{m} \varphi \mathrm{d} x \mathrm{~d} t-\int_{\mathcal{P}_{\delta}} \mathrm{d} \mu(\rho) \iint_{Q}\left(\bar{w}_{m}\right)_{\rho} \bar{u}_{m} \varphi \mathrm{d} x \mathrm{~d} t\right| \\
& \quad \leqslant\left|\int_{\mathcal{P} \backslash \mathcal{P}_{\delta}} \mathrm{d} \mu(\rho) \iint_{Q}\right| \bar{u}_{m}|\varphi \mathrm{d} x \mathrm{~d} t| \leqslant \mu\left(\mathcal{P} \backslash \mathcal{P}_{\delta}\right)\left\|\bar{u}_{m}\right\|_{L^{1}(Q)} \max _{Q \times \mathcal{P}} \varphi \rightarrow 0 \quad \text { as } \varepsilon \rightarrow 0
\end{aligned}
$$

uniformly in $m$. Therefore (7.18) yields (7.15).

Remarks. - (i) If $F \in L^{1}\left(0, T ; L^{2}(\Omega)\right)+W^{1,1}\left(0, T ; H^{-1}(\Omega)\right)$, the formulation of Problems 1 and 2 and the corresponding existence results are easily extended.

(ii) Theorems 5.3 and 7.2 can be extended if $-\Delta$ is replaced by any (nondegenerate) self-adjoint, second order, elliptic operator in divergence form with constant coefficients. The same applies if terms like $\frac{\partial u}{\partial t}$ or $\frac{\partial w}{\partial t}$ are included in the hyperbolic equation.

\section{A mean field model}

A class of parabolic equations. The representation of the relay operator based on the confinement condition (3.6) and on the dissipation inequality (3.9) can also be used to deal with quasilinear parabolic equations with hysteresis of the form

$$
\frac{\partial}{\partial t}\left[u+\mathcal{F}_{\mu}(u)\right]+A u=f .
$$

The eddy-current Eq. (2.7) is an example of this class. Existence of a solution for a corresponding initial- and boundary-vale problem can be proved via approximation, derivation of a priori estimates, passage to the limit. Here the main estimates are derived multiplying the approximate equation by the approximate $u$; this applies also if $A$ is not self-adjoint. See also Problem 5 below. If $A$ is self-adjoint, stronger regularity results can be proved multiplying the approximate equation by the approximate $\frac{\partial u}{\partial t}$; see, e.g., [10, Chap. IX].

A regularity issue. The formulation of Problem 1 rests on two issues:

(i) the use of the completed relay operators, $k_{\rho}$, in place of the standard relay operators, $h_{\rho}$;

(ii) the representation of the condition (3.5) via the inequality (4.5); on account of Eq. (4.6), this inequality is formally equivalent to (4.10). The latter formula would be meaningful only if one were able to give a meaning to $\int_{0}^{t} H^{-1}(\Omega)\langle\partial(u+$ $w) / \partial \tau, u\rangle_{H_{0}^{1}(\Omega)} \mathrm{d} \tau$, or to some other duality product of the same functions 
over $Q_{t}$. Notice that $\partial w / \partial \tau$ is just a Borel measure over $\bar{Q}$, and $u$ can hardly be expected to be an element of $L^{2}\left(0, T ; H_{0}^{1}(\Omega)\right)$ or to be continuous in $\bar{Q}$. Anyway this shortcoming occurs neither for the ODE

$$
\left.\frac{d^{2}}{\mathrm{~d} t^{2}}(u+w)+a u=f, \quad w \in k_{\rho}(u) \text { in }\right] 0, T[(a: \text { constant }>0),
$$

nor for the parabolic Eq. (8.1) in a single dimension of space, if $A$ is selfadjoint. In the latter case indeed, by multiplying the approximate equation by the approximate $\partial u / \partial t$, one can derive a uniform bound for the approximate $u$ in $H^{1}\left(0, T ; L^{2}(\Omega)\right) \cap L^{\infty}\left(0, T ; H_{0}^{1}(\Omega)\right)$, and the latter space is compactly imbedded in $C^{0}(\bar{Q})$, for $N=1$.

Concerning equations including the Preisach operator $\mathcal{F}_{\mu}$, if this operates in $C^{0}([0, T])$ then it is equivalent to deal with $k_{\rho}$ or with the originary relays, $h_{\rho}$. In the other cases the extension (i) is actually needed. Indeed, even for the Cauchy problem for the ODE (8.2) one can easily construct a function $f$ for which the only solution is such that $w$ does attain values in $]-1,1[$.

A mean field model. The above regularity problems are removed if Eq. (1.1) is coupled with a relation of the form

$$
w=\mathcal{F}_{\mu}(u * \eta) * \eta \quad \text { in } Q .
$$

Here $\eta$ is a bell-shaped mollifier, for instance,

$$
\eta(x):=(\pi \lambda)^{-3 / 2} \exp \left(-\frac{|x|^{2}}{\lambda}\right) \quad \forall x \in \mathbf{R}^{3}(\lambda: \text { constant }>0) ;
$$

by $*$ we denote the convolution in space, $u * \eta(x):=\int_{\mathbf{R}^{3}} u(x-y) \eta(y) \mathrm{d} y$ for any $x \in \Omega$. Prior to convolution, here $u$ has been extended with value 0 outside $\Omega$. The transformation $u \mapsto u * \eta$ may be interpreted as a length-scale transformation from mesoscopic to macroscopic variables; (8.3) then represents a mean field hysteresis relation.

Let us assume that

$$
\begin{aligned}
& u^{0}, z^{0} \in L^{2}(\Omega), \quad F \in L^{\infty}\left(0, T ; H^{-1}(\Omega)\right), \\
& \left|z^{0}\right| \leqslant 1, \quad z^{0}=-1 \text { if } u^{0}<\rho_{1}, \quad z^{0}=1 \text { if } u^{0}>\rho_{2} \quad \text { a.e. in } \Omega,
\end{aligned}
$$

fix any $a>0$, set $X_{t}:=C^{0}\left([0, t] ; H^{-a}(\Omega)\right)$ for any $t>0$, and define $\int_{\bar{\Omega}} \Psi_{\rho}(\cdot, t)$ as in (3.12).

We can now provide a weak formulation of the initial- and boundary-value problem associated with (1.1) and (8.3). For the sake of simplicity, here we just deal with a single completed relay, i.e., we assume that $\mu$ is a Dirac mass concentrated at a point of the Preisach plane.

Problem 3. - To find $U \in W^{1, \infty}\left(0, T ; L^{2}(\Omega)\right) \cap L^{\infty}\left(0, T ; H_{0}^{1}(\Omega)\right)$ and $z \in L^{\infty}(Q)$ such that $\gamma_{0} U=0$ a.e. in $(\Omega \times\{0\}) \cup(\partial \Omega \times] 0, T[),|z| \leqslant 1$ a.e. in $Q$, and $\partial z / \partial t \in$ 
$C^{0}(\bar{Q})^{\prime}$. Moreover, we set

$$
u:=\frac{\partial U}{\partial t}, \quad w:=z * \eta \text { a.e. in } Q, \quad z(\cdot, 0):=z^{0}, \quad w^{0}:=z^{0} * \eta \quad \text { a.e. in } \Omega,
$$

and require that

$$
\begin{aligned}
& \iint_{Q}\left[\left(u^{0}+w^{0}-u-w\right) \frac{\partial \eta}{\partial t}+\nabla U \cdot \nabla \eta\right] \mathrm{d} x \mathrm{~d} t=\int_{0}^{T}\langle F, \eta\rangle \mathrm{d} t \\
& \forall \eta \in C^{1}(\bar{Q}), \eta=0 \text { a.e. in }(\Omega \times\{T\}) \cup(\partial \Omega \times] 0, T[), \\
& (z-1)\left(u * \eta-\rho_{2}\right) \geqslant 0, \quad(z+1)\left(u * \eta-\rho_{1}\right) \geqslant 0 \quad \text { a.e.in } Q, \\
& \quad\left\langle\frac{\partial w}{\partial \tau}, u+w\right\rangle_{X_{t}} \geqslant \frac{1}{2} \int_{\Omega}\left[w(x, t)^{2}-w^{0}(x)^{2}\right] \mathrm{d} x+\int_{\bar{\Omega}} \Psi_{\rho}(z, t) \\
& \quad \text { for a.a.t } t \in] 0, T[.
\end{aligned}
$$

Interpretation. In Section 4 we saw that (8.6) is equivalent to the system (4.6) and (4.7). By the regularity we assumed for $U$ and $z$, we have $u+w \in L^{\infty}\left(0, T ; L^{2}(\Omega)\right)$; moreover a comparison in (4.6) yields $u+w \in W^{1, \infty}\left(0, T ; H^{-1}(\Omega)\right)$. Hence $u+w \in X_{t}$ for any $t \in] 0, T$, by a generalization of the Ascoli theorem. As $\frac{\partial w}{\partial \tau}=\frac{\partial z}{\partial \tau} * \eta \in X_{t}^{\prime}$, the duality pairing that occurs in (8.8) is meaningful. As

$$
\begin{aligned}
\int_{\Omega} \mathrm{d} \tau \int_{\Omega} \frac{\partial z}{\partial \tau} u * \eta \mathrm{d} x & =\int_{\Omega} \mathrm{d} \tau \int_{\Omega} \frac{\partial w}{\partial \tau} u \mathrm{~d} x \\
& =\int_{\Omega} \mathrm{d} \tau \int_{\Omega} \frac{\partial w}{\partial \tau}(u+w) \mathrm{d} x-\frac{1}{2} \int_{\Omega}\left[w(x, t)^{2}-w^{0}(x)^{2}\right] \mathrm{d} x,
\end{aligned}
$$

by (3.6) and (3.10), (8.7) and (8.8) represent the hysteresis relation

$$
z \in k_{\rho}\left(u * \eta, w^{0}\right), \quad \text { i.e., } \quad w \in k_{\rho}\left(u * \eta, w^{0}\right) * \eta \quad \text { a.e. in } \Omega .
$$

In conclusion, Problem 3 is a weak formulation of an initial- and boundary-value problem associated to the system (4.6) and (8.9).

THEOREM 8.1. - Under the hypothesis (4.1), Problem 3 has a solution.

The argument is similar to that of Theorem 5.3. Problem 3 and the above existence result can be extended to a general Preisach operator along the lines of Sections 6, 7.

Convergence to an equation without hysteresis. Let us now fix any $r \in \mathbf{R}$, and deal with the behaviour of the solution of Problem 3 as $\rho:=\left(\rho_{1}, \rho_{2}\right) \rightarrow(r, r)$.

Proposition 8.2. - For any $\rho \in \mathcal{P}$, there exists a solution $\left(U_{\rho}, w_{\rho}\right)$ of Problem 3 such that, as $\rho \rightarrow(r, r)$ along a suitable sequence,

$$
\begin{array}{ll}
U_{\rho} \rightarrow U & \text { weakly star in } W^{1, \infty}\left(0, T ; L^{2}(\Omega)\right) \cap L^{\infty}\left(0, T ; H_{0}^{1}(\Omega)\right), \\
w_{\rho} \rightarrow w & \text { weakly star in } L^{\infty}(Q) .
\end{array}
$$


Moreover $w:=z * \eta$, where $(U, z)$ is a suitable solution of the following reduced problem.

Problem 4. - To find $U \in W^{1, \infty}\left(0, T ; L^{2}(\Omega)\right) \cap L^{\infty}\left(0, T ; H_{0}^{1}(\Omega)\right)$ and $z \in L^{\infty}(Q)$ such that $\gamma_{0} U=0$ a.e. in $(\Omega \times\{0\}) \cup(\partial \Omega \times] 0, T[)$, and such that, setting

$$
\begin{gathered}
u:=\frac{\partial U}{\partial t}, \quad w:=z * \eta \quad \text { a.e. in } Q, \quad w^{0}:=z^{0} * \eta \quad \text { a.e. in } \Omega, \\
\iint_{Q}\left[\left(u^{0}+w^{0}-u-w\right) \frac{\partial \eta}{\partial t}+\nabla U \cdot \nabla \eta\right] \mathrm{d} x \mathrm{~d} t=\int_{0}^{T}\langle F, \eta\rangle \mathrm{d} t \\
\forall \eta \in C^{1}(\bar{Q}), \eta=0 \text { a.e. in }(\Omega \times\{T\}) \cup(\partial \Omega \times] 0, T[), \\
z \in \operatorname{sign}(u * \eta) \quad \text { a.e. in } Q .
\end{gathered}
$$

The argument is fairly standard. In particular, notice that in the limit we get

$$
(z-1)(u * \eta-r) \geqslant 0, \quad(z+1)(u * \eta-r) \geqslant 0 \quad \text { a.e. in } Q
$$

which is tantamount to (8.13). Therefore Problem 4 is an initial- and boundary-value problem for the inclusion

$$
\frac{\partial}{\partial t}\left[\frac{\partial U}{\partial t}+\operatorname{sign}\left(\frac{\partial U}{\partial t} * \eta\right) * \eta\right]-\Delta U=F \quad \text { in } Q .
$$

The latter result can also be extended to a general Preisach model; in this case in the limit one gets $z \in \alpha(u * \eta), \alpha$ being a bounded maximal monotone graph.

\section{Remarks and conclusions}

About the Preisach model. The Preisach half-plane $\mathcal{P}$ is open, i.e., it does not contain any point of the main diagonal $\left\{\rho \in \mathbf{R}^{2}: \rho_{1}=\rho_{2}\right\}$. Therefore the two curves that bound the major hysteresis loop can only merge along horizontal branches. For instance, the hysteresis relations outlined in Fig. 2 and in Fig. 5(a) can be represented in the form $w=\mathcal{F}_{\mu}(u), \mathcal{F}_{\mu}$ being a Preisach operator. This does not apply to Fig. 5(b), which can only represent a relation of the form $w=\mathcal{F}_{\mu}(u)+\varphi(u)$, for a suitable nonvanishing real function $f$. (Of course, the latter relation might also be represented by a Preisach operator if we allowed the support of $\mu$ to intersect the main diagonal. But other drawbacks would then arise.)

If the $w$ vs. $u$ relation is of the form represented in Fig. 5(b), Theorem 7.2 does not apply. However, DiPerna [3] proved existence of a solution for the equation

$$
\frac{\partial^{2}}{\partial t^{2}}[u+\varphi(u)]-\Delta u=f
$$




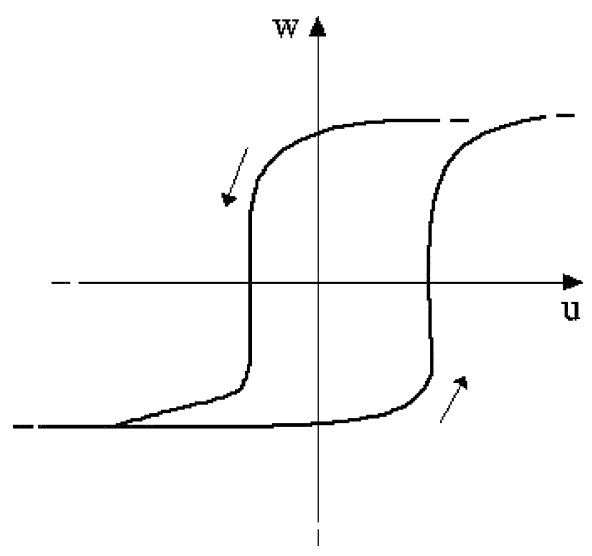

(a)

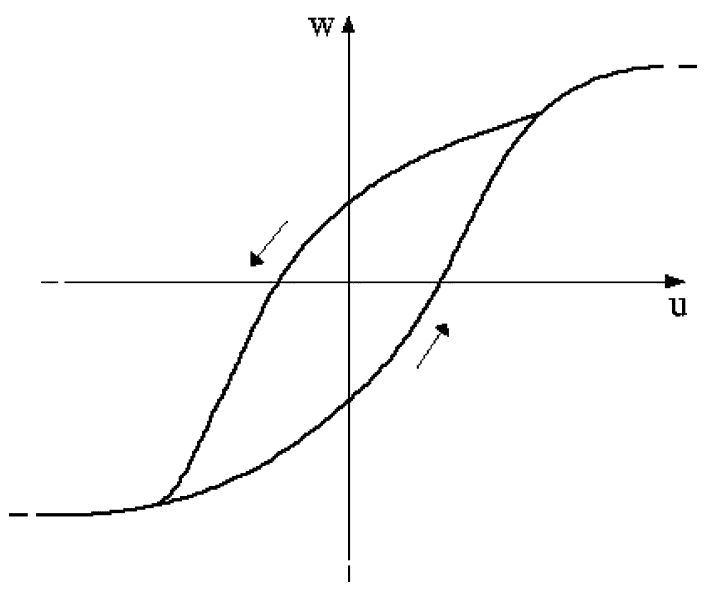

(b)

Fig. 5. Examples of hysteresis loops. Only the first one can be represented in the form $w=\mathcal{F}_{\mu}(u)$, for a Preisach operator $\mathcal{F}_{\mu}$.

for any monotone and strictly convex function $\varphi$. One might then try to prove existence of a solution for the equation

$$
\frac{\partial^{2}}{\partial t^{2}}\left[u+\mathcal{F}_{\mu}(u)+\varphi(u)\right]-\Delta u=f \quad \text { in } Q,
$$

by combining the above technique with that of DiPerna.

Other equations. (i) The existence results of Sections 5, 7 can easily be extended to degenerate hyperbolic equations of the form

$$
\frac{\partial^{2}}{\partial t^{2}} \mathcal{F}_{\mu}(u)+\frac{\partial u}{\partial t}+A u=f \quad \text { in } Q
$$

where $\mathcal{F}_{\mu}$ represents a Preisach operator. As it is easy to see, here one gets the regularity $U \in H^{1}\left(0, T ; L^{2}(\Omega)\right)$ in place of $U \in W^{1, \infty}\left(0, T ; L^{2}(\Omega)\right)$. If in Eq. (9.2) the term $\frac{\partial u}{\partial t}$ is dropped, existence of a solution is an open question.

(ii) As a further example, let us couple the degenerate quasilinear parabolic equation

$$
\frac{\partial}{\partial t} \mathcal{F}_{\mu}(u)+A u=f \quad \text { in } Q
$$

with an initial condition for $w:=\mathcal{F}_{\mu}(u)$ and with the homogeneous Dirichlet condition for $u$. For any $f \in L^{2}\left(0, T ; H^{-1}(\Omega)\right)$, we can formulate this problem as follows.

Problem 5. - To find $u \in L^{2}\left(0, T ; H_{0}^{1}(\Omega)\right)$ and $w \in L^{\infty}(Q \times \mathcal{P})$ such that $|w| \leqslant 1$ a.e. in $Q \times \mathcal{P}, \frac{\partial w}{\partial t} \in C^{0}(\bar{Q} \times \mathcal{P})^{\prime}$, and such that, setting $w(\cdot, 0):=w^{0}$ a.e. in $\Omega \times \mathcal{P}$, 


$$
\begin{gathered}
\iint_{Q}\left[\left(w^{0}-w\right) \frac{\partial \eta}{\partial t}+\nabla u \cdot \nabla \eta\right] \mathrm{d} x \mathrm{~d} t=\int_{0}^{T}\langle f, \eta\rangle \mathrm{d} t \\
\forall \eta \in C^{1}(\bar{Q}), \eta=0 \text { a.e. in }(\Omega \times\{T\}) \cup(\partial \Omega \times] 0, T[), \\
\left(w_{\rho}-1\right)\left(u-\rho_{2}\right) \geqslant 0, \quad\left(w_{\rho}+1\right)\left(u-\rho_{1}\right) \geqslant 0 \quad \text { a.e. in } Q \times \mathcal{P}, \\
\left.\int_{0}^{t} \mathrm{~d} \tau \int_{\Omega}|\nabla u(x, \tau)|^{2} \mathrm{~d} x+\iint_{\bar{\Omega} \times \mathcal{P}} \Psi_{\rho}\left(w_{\rho}, t\right) \leqslant \int_{0}^{t}\langle f, u\rangle \mathrm{d} \tau \quad \text { for a.a. } t \in\right] 0, T[.
\end{gathered}
$$

Existence of a solution can be proved by a technique similar to that of Section 5 . This formulation and the existence result can easily be extended to the Preisach model.

\section{Conclusions}

Several phenomena yield second order quasilinear hyperbolic equations with hysteresis of the form (1.1) for vector variables; in one-dimensional domains, the latter are reduced to scalars. However our analysis technique applies to scalar equations in domains of any dimension. It also allows for discontinuous hysteresis relations, thus for the occurrence of free boundaries.

We provided a weak formulation of an initial- and boundary-value problem for (1.1) in the framework of Sobolev spaces, at first for $\mathcal{F}$ equal to a relay operator, then for the Preisach model. For these problems we proved existence of a solution via approximation, derivation of a priori estimates, passage to the limit. The dissipative character of hysteresis provided a uniform estimate for $\partial w_{m} / \partial t$ in $L^{1}(Q)$; this allowed us to pass to the limit in the hysteresis relation, via a compensated compactness argument.

We took profit of occurrence of hysteresis to prove stronger results than are known for the corresponding problem without hysteresis. The equation with hysteresis can then be used to approximate that without hysteresis; however, if one lets the hysteresis effect vanish (and thus lets the hysteresis loop degenerate into a curve), then the typical difficulties of quasilinear hyperbolic equations are retrieved.

The analysis of tensor models of elastoplasticity and of vector models of ferromagnetism are major issues. For the relevant class of Prandtl-Ishlinskii models of elastoplasticity, $\mathcal{F}$ can be represented by a system of variational inequalities. In this case the analysis of our problem is fairly well understood, see [10, Chaps. III, VII]. (However, an extension of the Preisach model to rank-two tensors is not a priori excluded.)

The study of vector ferromagnetism looks more challenging; here even the formulation of the vector hysteresis relation is not completely clear. In [12] the Maxwell equations have been coupled with a vector hysteresis model of [2], distinguishing the cases in which displacement currents are either included or neglected; existence of a solution has been proved for the respective hyperbolic and parabolic problems, by using techniques of the present paper.

The existence results we proved in this article are based on approximation by implicit time-discretization. This requires the minimization of a (finite) family of functionals; therefore this approximation procedure is also convenient for numeric implementation. 
For quasilinear parabolic problems with Preisach hysteresis, it is known that the solution is unique and depends continuously on the data. On the other hand, for hyperbolic problems the uniqueness of the solution is largely an open question. However, for one-dimensional systems, Krejčí proved uniqueness in a more restricted class than that in which we have existence of a solution, see, [6, Section III.2]. Open questions also include the existence of periodic solutions, and the large-time behaviour of the solution(s), too.

\section{REFERENCES}

[1] Brokate M., Sprekels J., Hysteresis and Phase Transitions, Springer, Berlin, 1996.

[2] Damlamian A., Visintin A., Une généralisation vectorielle du modèle de Preisach pour l'hystérésis, C. R. Acad. Sci. Paris Série I 297 (1983) 437-440.

[3] DiPerna R.J., Convergence of approximate solutions of conservation laws, Arch. Rational Mech. Anal. 82 (1983) 27-70.

[4] Krasnosel'skiı̌ M.A., Pokrovskiı̌ A.V., Systems with Hysteresis, Springer, Berlin, 1989 (Russian ed.: Nauka, Moscow, 1983).

[5] Krejčí P., Hysteresis and periodic solutions of semi-linear and quasi-linear wave equations, Math. Z. 193 (1986) 247-264.

[6] Krejčí P., Convexity, Hysteresis and Dissipation in Hyperbolic Equations, Gakkotosho, Tokyo, 1997.

[7] Lions J.L., Magenes E., Non-Homogeneous Boundary Value Problems and Applications, Vols. I, II, Springer, Berlin, 1972 (French ed.: Dunod, Paris, 1968).

[8] Preisach F., Über die magnetische Nachwirkung, Z. Physik 94 (1935) 277-302.

[9] Triebel H., Interpolation Theory, Function Spaces, Differential Operators, Verlag der Wissenschaften, Berlin, 1978.

[10] Visintin A., Differential Models of Hysteresis, Springer, Berlin, 1994.

[11] Visintin A., Forward-backward parabolic equations and hysteresis, Differential and Integral Equations 9 (2000) 64-69.

[12] Visintin A., Maxwell's equations with vector hysteresis, Journal of Material Processing and Manufacturing Science (to appear).

[13] Visintin A., Hyperbolic equations with hysteresis, C. R. Acad. Sc. Paris, Série I 332 (2001) 315-320.

[14] Visintin A., Maxwell equations with vector hysteresis, in preparation. 